\title{
Larger urethral catheter size leads to fossa navicularis stricture formation in robotic radical prostatectomy
}

\author{
David S. Yee · Joel Gelman · Douglas W. Skarecky • \\ Thomas E. Ahlering
}

Received: 20 December 2006 / Accepted: 21 March 2007 / Published online: 27 April 2007

(C) Springer London 2007

\begin{abstract}
Fossa navicularis strictures following radical prostatectomy are reported infrequently. We recently experienced a series of fossa strictures following roboticassisted laparoscopic prostatectomy (RLP). We describe herein our experience to prevent fossa strictures and to determine its etiologic factors. From June 2002 to May 2006, 424 patients underwent robotic-assisted laparoscopic prostatectomy with the da Vinci surgical system. Fossa strictures were diagnosed based on the acute onset of obstructive voiding symptoms and bougie calibration. During our series, we switched from the intra-operative use of an 18 French $(\mathrm{F})$ catheter to that of a $22 \mathrm{~F}$ one to avoid inadvertent stapling of the urethra when dividing the dorsal venous complex. After we observed a high incidence of fossa strictures, we reverted back to $18 \mathrm{~F}$ catheters during surgery. All patients had an $18 \mathrm{~F}$ catheter indwelling for 1 week after surgery. Parameters were evaluated using Fisher's exact test and Student's $t$-test for means. The $18 \mathrm{~F}$ catheter group of patients $(n=293)$ developed one fossa stricture, whereas the $22 \mathrm{~F}$ catheter group $(n=131)$ developed nine fossa strictures $(P<0.01)$. The fossa stricture rate in the $18 \mathrm{~F}$ group was $0.3 \%$ versus $6.9 \%$ in the $22 \mathrm{~F}$ group. The two groups had no differences in age, body
\end{abstract}

Statement of disclosure Dr. Thomas Ahlering is a meeting participant and lecturer for Intuitive Surgical Corp. The other authors have no direct or indirect commercial financial incentives associated with publishing the article. No research or project support funding was received.

D. S. Yee $(\bowtie) \cdot$ J. Gelman · D. W. Skarecky · T. E. Ahlering Irvine Medical Center, University of California, 101 The City Drive, Bldg 26, Rm 204, Route 81, Irvine, CA 92868, USA

e-mail: dyee@uci.edu mass index, cardiovascular disease, American Urological Association symptom score, urinary bother score, preoperative prostate-specific antigen, operative time, estimated blood loss, cautery use, prostate size, or catheterization time. Based on these results, a larger urethral catheter size $20 \mathrm{~F}$ versus $18 \mathrm{~F}$ - during the intra-operative dissection would appear to increase the risk for fossa stricture by more than 20 -fold.

Keywords Laparoscopy · Robotic-assisted surgery · Robotics · Urethral stricture

\section{Introduction}

Fossa navicularis strictures following radical prostatectomy are rare. Unlike other anterior urethral strictures, fossa strictures are usually inflammatory or procedure-induced, arising from urethral trauma caused by endoscopic procedures, catheterizations, or subsequent infections [1]. In the literature, catheter size has not been described as a contributory factor. We describe the incidence of fossa stricture in our RLP series to determine its likely etiologic factors.

\section{Material and methods}

The data of 424 men undergoing RLP from June 2002 to May 2006 by a single surgeon were prospectively entered into an electronic database. Prior to surgery, all men were evaluated, and the following data entered into the database: age, height, weight, American Urological Association (AUA) symptom score, urinary bother score, prostate-specific antigen (PSA), and pertinent medical history. Standard perioperative and postoperative parameters were evaluated. 
Urinary and functional outcomes were determined on the basis of self-administered questionnaires, including the seven-item International Prostate Symptom Score, and selected questions from the 26-item Expanded Prostate Cancer Instrument Composite, at the routine 3-month and 9-month follow-up visits. The questionnaire asked whether they wore pads, how many weeks did it take to not need pads, how many weeks needed to return to work, and how many weeks to return to baseline energy levels. A nonclinical research associate (D.W.S.) collected the follow-up information. Complications were defined by the need for prolongation of hospitalization, the need for a secondary procedure, or rehospitalization within 30 days. All statistical comparisons between the stricture and nonstricture groups as well as between the 18 and 22 French $(F)$ catheter groups were two-sided using Fisher's exact test and Student's $t$-test for means (SAS ver. 8.2, statistical package; SAS, Cary, N.C.). Multivariate analysis was also performed with a stepwise logistic regression which used the preoperative continuous variables, body mass index (BMI), PSA, estimated blood loss (EBL), AUA symptom score, bother score, age, and prostate weight (Table 1) as independent variables in the prediction of a positive stricture. Ongoing institutional review board approval has been in place since 1998.

All RLPs were performed in the same manner with the da Vinci surgical system (Intuitive Surgical, Mountain View, Calif.). Patients are positioned supine in an extreme Trendelenburg position and undergo abdominal insufflation with a pneumoperitoneum of $15 \mathrm{mmHg}$. The standard protocol consists of inserting a silastic urethral catheter at the beginning of the case that is utilized throughout the dissection until completion of the anastomosis. At this point a new $18 \mathrm{~F}$ silastic catheter is put in place, and this is generally removed 7 days postoperatively. At case number 35 , however, we switched from an 18 to a $22 \mathrm{~F}$ silastic urethral catheter during the dissection as an aid to prevent stapling the urethra. We use a stapling device instead of suturing the dorsal venous complex based on findings from a previous study. In that study, the data demonstrated that the stapler technique provided a more defined apical dissection and a statistically significant reduction in positive margins in patients with organ-confined disease [2]. We switched back to an $18 \mathrm{~F}$ catheter at case number 166 due to concerns regarding strictures in the fossa navicularis.

Fossa strictures were diagnosed based on acute onset of obstructive lower urinary symptoms and bougie a boule calibration. Obstructive voiding symptoms included a decreased force of stream, dribbling or splaying, and prolonged voiding.

\section{Results}

Table 1 presents the demographic, clinical, and perioperative data for men reporting strictures versus stricture-free men. The mean follow-up was 26.4 months for stricture patients versus 22.1 months for stricture-free patients and 22.8 months (range: 3.6-45.6 months) for all patients. The groups were comparable for the standard clinical factors, such as age, BMI, AUA symptom score, urinary bother score, and preoperative PSA level. There was also no difference between the groups in reference to operative time, prostate size, and cardiovascular disease. The stricture-free group, however, had a higher EBL than the stricture group $(P=0.03)$. In addition, none of the patients in the stricture group had any history of a previous endoscopic procedure. Multivariate analysis also demonstrated that none of the preoperative continuous variables predicted the presence of strictures (all $P>0.18$ ).

Table 2 outlines the demographic, clinical, and perioperative data for men stratified by catheter size. The 18 and 22 F catheter groups were also comparable for age, BMI, baseline AUA symptom score, urinary bother score, preoperative

Table 1 Demographic, clinical, and perioperative data for men reporting strictures versus stricture-free men

\begin{tabular}{|c|c|c|c|c|c|}
\hline Variable $^{\mathrm{a}}$ & No stricture ${ }^{b}$ & Standard error (SE) & Stricture $^{\mathrm{b}}$ & SE & $P$-value \\
\hline Patients $(n)$ & 414 & & 10 & & \\
\hline Age (year) & $61.4 .0(43-79)$ & 0.3 & $63.8(51-71)$ & 2.4 & 0.29 \\
\hline BMI & $27.0(20.6-34.9)$ & 0.2 & $26.3(24.2-34.0)$ & 0.7 & 0.52 \\
\hline AUA symptom score & $8.6(0-32)$ & 0.4 & $7.4(1-27)$ & 1.8 & 0.59 \\
\hline Urinary bother score & $1.7(0-6)$ & 0.1 & $1.6(0-6)$ & 0.5 & 0.86 \\
\hline Preoperative PSA (ng/ml) & $6.9(0.1-62.0)$ & 0.3 & $6.2(1.8-18.0)$ & 1.5 & 0.71 \\
\hline Prostate size $(\mathrm{g})$ & $53.1(12.5-163.0)$ & 1.4 & $46.1(20.0-69.1)$ & 3.3 & 0.41 \\
\hline $\mathrm{EBL}(\mathrm{ml})$ & $107(25-350)$ & 2.8 & $67.5(25-400)$ & 16.3 & $0.03^{\mathrm{c}}$ \\
\hline
\end{tabular}

a BMI, Body mass index; AUA, American Urological Association; PSA, prostate-specific antigen; EBL, estimated blood loss

b Values are the mean, with the range given in parenthesis

c Two-sided Fisher's exact test 
Table 2 Demographic, clinical data, and perioperative data for 18 and $22 \mathrm{~F}$ catheter groups

\begin{tabular}{|c|c|c|c|c|c|}
\hline Variable $^{a}$ & $18 \mathrm{~F}^{\mathrm{b}}$ & SE & $22 \mathrm{~F}^{\mathrm{b}}$ & SE & $P$-value \\
\hline Patients (n) & 293 & & 131 & & \\
\hline Age (year) & $61.1(46-79)$ & 0.4 & $62.2(43-78)$ & 0.7 & 0.14 \\
\hline BMI & $27.0(20.6-40.0)$ & 0.2 & 26.8 & 0.3 & 0.49 \\
\hline AUA symptom score & $8.7(0-35)$ & 0.4 & $8.2(0-31)$ & 0.6 & 0.54 \\
\hline Urinary bother score & $1.7(0-6)$ & 0.1 & $1.7(0-6)$ & 0.1 & 0.93 \\
\hline Preoperative PSA (ng/ml) & $6.7(1.1-31.0)$ & 0.3 & $7.3(0.1-62.0)$ & 0.6 & 0.30 \\
\hline Prostate size $(\mathrm{g})$ & $54.1(12.5-163.0)$ & 1.8 & $50.5(15.0-135.0)$ & 1.9 & 0.21 \\
\hline $\mathrm{EBL}(\mathrm{ml})$ & $109.3(25-350)$ & 3.2 & $98.9(25-400)$ & 5.5 & 0.10 \\
\hline Fossa stricture & 1 & & 9 & & $0.0002^{c}$ \\
\hline
\end{tabular}

${ }^{a}$ BMI, Body mass index; AUA, American Urological Association; PSA, prostate-specific antigen; EBL estimated blood loss

${ }^{b}$ Values are the mean, with the range given in parenthesis

c Two-sided Fisher's exact test

PSA, operative time, prostate size, EBL, and complications. The $22 \mathrm{~F}$ catheter group, however, had a significantly higher rate of stricture formation. A total of $6.9 \%$ of patients developed a stricture using a $22 \mathrm{~F}$ catheter versus $0.3 \%$ of patients using an $18 \mathrm{~F}$ catheter during the intraoperative dissection $(P<0.01)$. The mean length of urethral catheterization was 7.1 days (range: $7-8$ days) in the stricture group, with a mean time to stricture development of 50 days (range: $34-93$ days). One patient in the stricture group developed a bladder neck contracture and another went into acute urinary retention.

In addition, urethral reconstruction was required in two of nine patients (22\%) who developed strictures with the larger $22 \mathrm{~F}$ catheter. Only one subject developed a stricture using an $18 \mathrm{~F}$ catheter; he required only soft dilation as treatment. In the present series, $76 \%$ of the RLP patients attained pad-free continence at 3 months. All comparisons made by the Student's $t$-test were also examined using the nonparametric Wilcoxon rank sum test, with similar conclusions.

\section{Discussion}

A thorough review of the literature revealed an absence of papers specifically focused on fossa strictures; most information relating to these strictures are a secondary thought to more common strictures of the bulbar urethra. It has been reported that, in general, strictures involving the fossa have three distinct etiologic factors: inflammatory, procedurerelated, or catheter-induced. Inflammatory conditions include balanitis xerotica obliterans (BXO), lichen sclerosis, and an inflammatory variant of vitiligo [1,3-6]. Procedure-related causes are most commonly associated with transurethral resection of the prostate, which has a reported rate of approximately $2.6 \%$ [7]. However, we could find no information suggesting that a larger caliber resectoscope was responsible for a greater risk of fossa strictures.

Catheter-induced strictures have been evaluated fairly extensively [8]. The incidence of urethral stricture following cardiovascular surgery in the early 1980s prompted much investigation and was thought to arise from catheter toxicity and urethral ischemia $[9,10]$. However, these strictures usually affected the anterior urethra and none solely involved the fossa navicularis. Abdel-Hakim and Elhilali $[11,12]$ found some evidence for urethral ischemia in stricture cases utilizing strain gauge plethysmography to determine penile-brachial indexes. In addition, Nacey [13] demonstrated a significantly increased incidence of urethral strictures following catheterization with silastic catheters compared to silicone catheters in a controlled randomized prospective study of patients undergoing elective cardiac surgery. Other experimental and clinical studies demonstrated that latex catheters were more toxic than non-latex catheters [14-18].

After a review of our first 100 cases, we noted five fossa strictures. As noted above, there were essentially no published reports of fossa strictures following RLP and very little information regarding its etiology. We initially looked at factors such as age, medical diseases, BMI, EBL, operative length, among others. for an etiology. Although EBL was noted to be significantly higher in stricture-free men, its association with stricture formation is unclear since the EBL between the two different catheter groups was essentially the same. With no obvious factor identified, we evaluated the use of monopolar cautery. We were concerned that the electrical current leaving the monopolar tip might potentially be transmitted down the catheter in the urethra and injure the fossa. In response to this potential source of injury, the grounding pad was moved from the upper thigh (i.e., adjacent to the penis) to the thorax. However, the fossa strictures continued. We 
next turned to the possibility that the $22 \mathrm{~F}$ caliber of the urethral catheter might be responsible. At case number 35 we had switched from our standard $18 \mathrm{~F}$ catheter to a $22 \mathrm{~F}$ caliber in an attempt to avoid inadvertent stapling of the urethra. At case number 166 we switched back. Remarkably, our data suggest - and continued experience supports the data - that the $22 \mathrm{~F}$ silastic urethral catheter is the likely etiology. The use of a $22 \mathrm{~F}$ silastic urethral catheter during the intra-operative dissection would appear to have increased the risk for fossa stricture 20 -fold relative to the $18 \mathrm{~F}$ catheter. However, definitive empirical proof that the larger sized catheter is the etiological factor is absent; it may be that in addition to catheter size, the combination of extreme Trendelenburg position and the pneumoperitoneum associated with RLP are other contributing factors. This combination of factors may account for the lack of reports on similar stricture experiences among the large volume of articles published on RLP and cystectomy.

The mean time to fossa stricture formation was $\mathbf{5 0}$ days (range: 34-93 days). The strictures were not equally distributed over time, with the majority of strictures diagnosed by postoperative day 50 . We do not have an explanation why some men developed strictures after one month and others after 3 months. As Table 1 demonstrates, there were no other obvious differences between the two groups. Two of ten strictures (both in the $22 \mathrm{~F}$ group) subsequently required open reconstruction, whereas $80 \%$ resolved with soft dilations continued over a 3- to 6-month interval following the development of the fossa stricture.

\section{Conclusion}

In summary, an $18 \mathrm{~F}$ catheter is sufficient to drain the bladder safely. A larger urethral catheter size during the intraoperative dissection in RLP appears to increase the risk for fossa navicularis stricture.

\section{References}

1. Armenakas NA, McAninch JW (2002) Management of fossa navicularis strictures. Urol Clin N Am 29:477-484

2. Ahlering TE, Eichel L, Edwards RA et al. (2004) Robotic radical prostatectomy: a technique to reduce pT2 positive margins. Urology 64:1224-1228

3. Khezri AA, Dounis A, Dunn M (1979) Balanitis xerotica obliterans. Br J Urol 51:229-231

4. Mall N, Garat JM, Santaularia J et al. (1978) Urethro-balanitis xerotica obliterans. Eur Urol 4:9-12

5. Staff WG (1970) Urethral involvement in balanitis xerotica obliterans. Br J Urol 47:234-239

6. Barbagli G, Palminteri E, Lazzeri M et al. (2001) Lichen sclerosis of the male genitalia. Contemp Urol 13:47-50

7. Lentz HC Jr, Mebust WK, Foret JD et al. (1977) Urethral strictures following transurethral prostatectomy: review of 2,223 resections. J Urol 117:194-196

8. Edwards L, Trott PA (1973) Catheter-induced urethral inflammation. J Urol 110:678-681

9. Ruutu M, Alfthan O, Heikkinen L et al. (1982) "Epidemic" of acute urethral stricture after open heart surgery. Lancet 1:218

10. Sutherland PD, Maddern JP, Jose JS et al. (1983) Urethral stricture after cardiac surgery. Br J Urol 55:413-416

11. Abdel-Hakim A, Hassouna M, Teijeira J et al. (1984) Role of urethral ischemia in the development of urethral strictures following cardiovascular surgery: a preliminary report. J Urol 131:10771079

12. Elhilali MM, Hassouna M, Abdel-Hakim A et al. (1986) Urethral stricture following cardiovascular surgery: role of urethral ischemia. J Urol 135:275-277

13. Nacey JN, Tulloch AGS, Ferguson AF (1985) Catheter induced urethritis: a comparison between silastic and silicone catheters in a prospective clinical trial. Br J Urol 57:325-328

14. Engelbart RH, Bartone FF, Gardner P et al. (1978) Urethral reaction to catheter materials in dogs. Invest Urol 16:55-56

15. Graham DT, Mark GE, Pomeroy AR (1983) Cellular toxicity of urinary catheters. Med J Aust 1:456-459

16. Keitzer WA, Abreu A, Navarro I et al. (1968) Urethral strictures: prevention with plastic indwelling catheters. J Urol 99:187-188

17. Painter MR, Borski AA, Trevino GS et al. (1971) Urethral reaction to foreign objects. J Urol 106:227-230

18. Engel RME, Wise HA, Whitaker RH (1972) Otis internal urethrotomy with long term urethral intubation: a comparison of latex and silastic catheters. South Med J 65:55-60 